\title{
Five-year Survival of Patients with AIDS Receiving Antiretroviral Therapy in Haiti
}

\author{
Paul Leger, MD1, Macarthur Charles, MD, Ph.D ${ }^{1,2}$, Patrice Severe, MD¹, Cynthia Riviere, \\ MD ${ }^{1}$, Jean William Pape, $\mathbf{M D}^{1,2}$, and Daniel W. Fitzgerald, $\mathbf{M D}^{1,2}$ \\ ${ }^{1}$ Groupe Haitien d'Etude du Sarcome de Kaposi et des Infections Opportunistes (GHESKIO), Port \\ au Prince, Haiti \\ ${ }^{2}$ Division of International Medicine and Infectious Diseases, Weill Cornell Medical College, New \\ York, NY \\ ${ }^{3}$ Division of Social Medicine and Health Inequalities, Brigham and Women's Hospital, Boston, MA
}

We previously reported antiretroviral therapy (ART) outcomes of adults with AIDS in Haiti with $87 \%$ survival at one year. ${ }^{1}$ We now report the 5-year outcomes of these 910 consecutive patients $\geq 13$ years of age who initiated ART according to World Health Organization (WHO) guidelines between March 2003 and April 2004 and who were followed at the GHESKIO clinic in Port au Prince, Haiti through May 2008. ${ }^{2}$

Of the 910 patients, 73 (8\%) were lost to follow-up, and 198 (22\%) died. For 738 patients in care at 6 months, $587(80 \%)$ had an adherence level $\geq 90 \%$. By Kaplan Meyer analysis, $89 \%$ of patients were alive at 6 months, $87 \%$ at 12 months, and $78 \%$ at 60 months. The mortality in the first six months ( 25 deaths/100 person-years) was seven times greater than the mortality after six months (3.6 deaths/100 person-years) $(\mathrm{p}<.0001)$. Death in the first six months of therapy was associated with having an AIDS defining illness $(\mathrm{p}<.0001)$, weight in the lowest quartile ( $\mathrm{p}<.0001)$, and a CD4 T cell count $<50$ cells $/ \mathrm{ml}(\mathrm{p}<.0001)$. Death after 6 months was associated with adherence $<90 \%$, $(\mathrm{p}<.0001)$, age $>50$ years $(\mathrm{p}$ $=.0009)$, and a diagnosis of tuberculosis during the first six months of ART $(\mathrm{p}=.0189)$.

Two hundred and eleven patients met WHO clinical and/or immunologic criteria for "ART failure" as of January 2007, and $113(53.5 \%)$ had plasma HIV-1 RNA greater than 50 copies $/ \mathrm{ml}$. The positive predictive value of clinical criteria (WHO stage III or IV HIV related symptoms) for detectable HIV-1 RNA was only $48 \%$. The positive predictive value of immunologic criteria (decrease in CD4 T cell count by $50 \%$ or return to baseline value) was $77 \%$. HIV-1 reverse transcriptase genotyping was performed for 91 patients who had plasma HIV RNA levels greater than 1, 000 copies/ml, (Table).

This report documents the long-term sustainability of ART programs in resource-poor countries with excellent retention, adherence, and 78\% survival at five years. Programs should strive to identify and treat patients before advanced AIDS develops. Clinical criteria are poor predictors of virologic failure; CD4 T cell criteria are better. However, waiting to meet the CD4 T cell criteria may delay the recognition of virologic failure and result in the accumulation of HIV-1 drug resistance mutations. Virologic monitoring should be made available in resource poor settings, and access should be secured to second line agents, which are effective against HIV-1 with multiple drug resistance mutations.

Correspondence: Daniel W Fitzgerald, Division of International Medicine and Infectious Diseases, Weill Medical College of Cornell University, Room A-421, 1300 York Avenue, New York, NY, USA, Phone: (212) 746 -6320, Fax: (212) 746-8675

(dfitzgerald@gheskio.org). 


\section{Acknowledgments}

The project was supported in part by grants AI58257 from the National Institutes of Allergy and Infectious Diseases and TW006896, TW006901, and TW 00018 from the Fogarty International Center, and grants from the Global Fund Against AIDS, Malaria, and Tuberculosis and the President's Emergency Plan for AIDS Relief (PEPFAR).

\section{References}

1. Severe P, Leger P, Charles M, et al. Antiretroviral therapy in a thousand patients with AIDS in Haiti. N Engl J Med. 2005; 353:2325-34. [PubMed: 16319381]

2. World Health Organization. Scaling up Antiretroviral Therapy in resource-limited settings. World Health Organization; Geneva: 2004.

3. Liu TF, Shafer RW. Web resources for HIV type 1 genotypic-resistance test interpretation. Clin Infect Dis. 2006; 42(11):1608-18. [PubMed: 16652319]

4. Johnson VA, Brun-Vezinet F, Clotet B, et al. Update of the drug resistance mutations in HIV-1: 2007. Top HIV Med. 2007; 15(4):119-25. [PubMed: 17720996] 


\section{Table}

HIV-1 resistance mutations in 91 patients with plasma HIV-1 RNA level > 1,000 copies/ml while receiving antiretroviral therapy *

HIV-1 mutation

Any drug resistance mutation

Mutation conferring resistance to non- nucleoside reverse transcriptase inhibitors (NNRTI ${ }^{\dagger}$

Reverse transcriptase M184V mutation conferring resistance to lamivudine

Mutations to both NNRTI and lamivudine

Mutations other than M184V conferring resistance to nucleoside reverse transcriptase inhibitors

Any thymidine analogue mutation (TAM) ${ }^{\ddagger}$

Two or more TAMs

Mutations conferring resistance to lamivudine, other NRTIs, and NNRTIs
Number of patients (\%)

80 (88)

$76(84)$

62 (68)

$56(62)$

46 (51)

42 (46)

30 (33)

37 (41)

The HIV-1 polymerase gene was amplified by PCR and then sequenced on an automated system (Applied Biosystems). The resistance profiles to antiretroviral drugs were defined according to the Stanford University algorithms. ${ }^{3}$ Mutations at positions in the polymerase gene associated with antiretroviral resistance by the International AIDS Society-USA Drug Resistance Mutations Group were noted. ${ }^{4}$

${ }^{\dagger}$ NNRTI mutations included K103N (43 patients), G190A (12 patients), Y181C/I (13 patients). Two patients had $\geq 3$ NNRTI mutations.

${ }^{{ }^{*}}$ TAMs included M41L ((20 patients), D67N (8 patients), K70R (16 patients), L210W (2 patients), T215Y/F (29 patients), K219Q/E (12 patients). 